\title{
A sustained systemic inflammatory response syndrome is associated with shunt-dependent hydrocephalus after aneurysmal subarachnoid hemorrhage
}

\author{
Aaron P. Wessell, MD, ${ }^{1}$ Matthew J. Kole, MD, ${ }^{1}$ Gregory Cannarsa, MD, ${ }^{1}$ Jeffrey Oliver, MD, ${ }^{1}$ \\ Gaurav Jindal, MD, ${ }^{1,4,6}$ Timothy Miller, MD, ${ }^{6}$ Dheeraj Gandhi, MBBS, ${ }^{1,4,6}$ Gunjan Parikh, MD, ${ }^{4}$ \\ Neeraj Badjatia, MD, MS, ${ }^{1,4,5}$ E. Francois Aldrich, MD, ${ }^{1}$ and J. Marc Simard, MD, PhD ${ }^{1-3}$ \\ Departments of ${ }^{1}$ Neurosurgery, ${ }^{2}$ Pathology, ${ }^{3}$ Physiology, ${ }^{4}$ Neurology, ${ }^{5}$ Anesthesiology, and ${ }^{6}$ Radiology, University of Maryland \\ School of Medicine, Baltimore, Maryland
}

\begin{abstract}
OBJECTIVE The authors sought to evaluate whether a sustained systemic inflammatory response was associated with shunt-dependent hydrocephalus following aneurysmal subarachnoid hemorrhage.

METHODS A retrospective analysis of 193 consecutive patients with aneurysmal subarachnoid hemorrhage was performed. Management of hydrocephalus followed a stepwise algorithm to determine the need for external CSF drainage and subsequent shunt placement. Systemic inflammatory response syndrome (SIRS) data were collected for all patients during the first 7 days of hospitalization. Patients who met the SIRS criteria every day for the first 7 days of hospitalization were considered as having a sustained SIRS. Univariate and multivariate regression analyses were used to determine predictors of shunt dependence.
\end{abstract}

RESULTS Sixteen percent of patients required shunt placement. Sustained SIRS was observed in $35 \%$ of shuntdependent patients compared to $14 \%$ in non-shunt-dependent patients $(p=0.004)$. On multivariate logistic regression, female sex (OR 0.35, 95\% Cl 0.142-0.885), moderate to severe vasospasm (OR 3.78, 95\% Cl 1.333-10.745), acute hydrocephalus (OR 21.39, 95\% Cl 2.260-202.417), and sustained SIRS (OR 2.94, 95\% Cl 1.125-7.689) were significantly associated with shunt dependence after aneurysmal subarachnoid hemorrhage. Receiver operating characteristic analysis revealed an area under the curve of 0.83 for the final regression model.

CONCLUSIONS Sustained SIRS was a predictor of shunt-dependent hydrocephalus following aneurysmal subarachnoid hemorrhage even after adjustment for potential confounding variables in a multivariate logistic regression model. https://thejns.org/doi/abs/10.3171/2018.1.JNS172925

KEYWORDS subarachnoid hemorrhage; cerebral aneurysm; inflammation; hydrocephalus; shunt; vascular disorders

$\mathrm{H}$ YDROCEPHALUS is a major source of morbidity following aneurysmal subarachnoid hemorrhage. As many as $6 \%-87 \%$ of patients develop acute hydrocephalus, while 6\%-64\% require permanent CSF diversion in the form of an implanted ventriculoperitoneal shunt or other shunt for the treatment of chronic hydrocephalus. 5,29

Traditionally, hydrocephalus following subarachnoid hemorrhage has been attributed to impaired CSF reab- sorption by arachnoid granulations, ventricular adhesions/ obstruction, and altered CSF dynamics. ${ }^{29}$ However, thrombin-induced transforming growth factor (TGF) $-\beta$ activity, nuclear factor (NF) $-\kappa B$ activation, and choroid plexus/ ependymal inflammation also have been associated with hydrocephalus. ${ }^{18,19,23}$

An inflammatory response, termed the systemic inflammatory response syndrome (SIRS), occurs in two-thirds of patients following subarachnoid hemorrhage. SIRS has

ABBREVIATIONS AUC = area under the curve; CTA = CT angiography; DND = delayed neurological deficit; $D S A=$ digital subtraction angiography; EVD = external ventricular drain; MFS = modified Fisher scale; NF = nuclear factor; ROC = receiver operating characteristic; SIRS = systemic inflammatory response syndrome; TGF = transforming growth factor; WFNS = World Federation of Neurosurgical Societies.

SUBMITTED November 19, 2017. ACCEPTED January 26, 2018.

INCLUDE WHEN CITING Published online June 29, 2018; DOI: 10.3171/2018.1.JNS172925. 
been associated with poor outcome independent of infection. ${ }^{4,33,44}$ To date, no study has evaluated the relationship between SIRS and shunt-dependent hydrocephalus after aneurysmal subarachnoid hemorrhage. In this study we tested the hypothesis that a sustained SIRS is associated with the development of shunt-dependent hydrocephalus following aneurysmal subarachnoid hemorrhage.

\section{Methods \\ Patient Selection}

The present study was conducted with the approval of the University of Maryland School of Medicine IRB. The records of a consecutive cohort of patients admitted to the University of Maryland Medical Center with aneurysmal subarachnoid hemorrhage from July 2013 to September 2016 were reviewed. Patients 18 years of age or older with subarachnoid hemorrhage, diagnosed by CT or xanthochromia on lumbar puncture, with a confirmed cerebral aneurysm on CT angiography (CTA) or digital subtraction angiography (DSA), were eligible for inclusion. We excluded patients who were not candidates for a shunt, including those who transitioned to palliative care, underwent withdrawal of care, or died during their hospital stay. Additionally, we excluded patients diagnosed with an infection of any kind during the first 7 days of hospitalization due to the potential confounding effect on the SIRS following subarachnoid hemorrhage. Infection was defined as a positive bacterial culture from blood, ${ }^{13}$ $\mathrm{CSF},{ }^{37}$ or clean-catch urine sample, ${ }^{12}$ or a positive Clostridium difficile assay on stool sample. ${ }^{2}$ Hospital-acquired or ventilator-associated pneumonias were diagnosed according to the clinical guidelines of the Infectious Disease Society of America. ${ }^{15}$

\section{Management of Patients With Aneurysmal Subarachnoid Hemorrhage}

Patients were managed according to the most recent Guidelines for the Management of Aneurysmal Subarachnoid Hemorrhage, published by the American Heart Association. ${ }^{3}$ An emphasis was placed on prompt aneurysm treatment with surgical clipping or endovascular coiling, treatment of acute hydrocephalus, maintenance of euvolemia, and treatment with oral nimodipine. Radiological studies such as CT, CTA, DSA, and MRA were routinely performed within 24 hours of treatment to confirm successful obliteration of the aneurysm and to assess for any complication of treatment or delayed development of hydrocephalus.

\section{Management of Hydrocephalus}

Our management of acute hydrocephalus followed an algorithm similar to that published previously. ${ }^{45}$ Both clinical and radiological features determined the decision to place an external ventricular drain (EVD), including clinical signs of hydrocephalus (confusion, lethargy/somnolence, or pupillary changes) associated with CT evidence of hydrocephalus (enlarging temporal horns, global ventriculomegaly, basilar cistern or sulcal effacement). ${ }^{24}$ In rare cases a lumbar drain was placed, although this is not the preferred method of CSF diversion at our institution.
The decision for shunt placement followed a similar algorithm performed in a stepwise fashion. ${ }^{24}$ Patients underwent continuous external drainage of CSF for treatment of acute hydrocephalus throughout their hospital courses. For patients who did not develop clinical signs of vasospasm, weaning of the EVD was begun on days 10-14 posthemorrhage. For patients who developed clinical vasospasm requiring hypertensive therapy and/or intraarterial therapy, EVD weaning commenced following resolution of clinically significant vasospasm. EVD weaning was executed in two phases. Initially, the patient was tested by gradually increasing the height of the external collection reservoir over several days to a maximum of $20 \mathrm{~cm} \mathrm{H}_{2} \mathrm{O}$. Subsequently, the EVD was clamped for 24-48 hours with serial clinical examinations, continuous intracranial pressure monitoring, and CT scans performed to monitor for the development of hydrocephalus. If the patient experienced a new neurological decline, or if intracranial pressure was sustained above $20 \mathrm{~mm} \mathrm{Hg}$ for $>10$ minutes, particularly in the setting of increasing ventricular size, the EVD was reopened. Patients who tolerated weaning the EVD but later showed clinical signs of hydrocephalus underwent a repeat CT scan or lumbar puncture to assess opening pressure. An opening pressure $<18 \mathrm{~cm} \mathrm{H}_{2} \mathrm{O}$ was considered acceptable. Patients who did not tolerate EVD weaning, demonstrated progressive ventriculomegaly on $\mathrm{CT}$, or had persistently elevated opening pressures on lumbar puncture underwent surgical placement of a shunt, typically a ventriculoperitoneal shunt.

\section{Data Compilation}

Patient records (including operative reports, radiological studies, hospital and clinic notes, and laboratory studies) were reviewed. Demographic, clinical, radiological, laboratory, and outcome data were compiled. Patients were graded clinically and radiologically according to the World Federation of Neurosurgical Societies (WFNS) grade and modified Fisher scale (MFS) score. ${ }^{6,35}$ In a few select cases in which these data were not initially recorded, WNFS grade and MFS score were determined retrospectively by two neurosurgeons. The occurrence of angiographic vasospasm was determined from independent review of CTA and DSA studies by neuroradiologists from the University of Maryland. Angiographic vasospasm was graded according to severity: mild (0\%-33\%), moderate $(34 \%-66 \%)$, or severe $(67 \%-100 \%)$ vessel narrowing in any vascular territory, independent of the patient's neurological status. ${ }^{41}$ The incidence of delayed neurological deficit (DND) was determined from review of inpatient records. DND was defined as a 2-point drop in Glasgow Coma Scale score or other neurological decline, such as a global change in mental status, pronator drift, or focal neurological deficit in the setting of confirmed angiographic vasospasm. In the absence of confirmed vasospasm, a decline meeting the above criteria with no other attributed medical causes was also considered a DND?

The occurrence of cerebral infarcts was determined from review of all CT and MR images by neurosurgeoninvestigators and independently by neuroradiologists from the University of Maryland. CT and MR images during hospitalization were correlated with angiographic studies 
to determine whether or not CT hypodensities or diffusionweighted imaging lesions on MRI were attributable to vasospasm (within a discrete vascular territory or watershed distribution), rather than resulting from intraparenchymal hemorrhage, EVD placement, surgical retraction, or the treatment-related loss of a perforating artery.

Discharge data were obtained from review of hospital records. Discharge disposition was defined as discharge to home or discharge to another location (including skilled nursing facility, and acute or subacute inpatient rehabilitation). Recommendations for discharge to home versus an inpatient nursing or rehabilitation facility were made independently by physical or occupational therapists based on patients' functional independence, as defined by the activities of daily living.

SIRS data were obtained from review of inpatient laboratory and ICU records. Patients were considered to have SIRS if they met two or more of the following SIRS criteria on any given day during the first 7 days of hospitalization: 1) temperature $>38^{\circ} \mathrm{C}$ or $<36^{\circ} \mathrm{C}$; 2) white blood cell count $>12,000$ or $<4000 ; 3$ ) heart rate $>90$ beats per minute (tachycardia); and 4) respiratory rate $>20$ breaths per minute (tachypnea).,33,44 Patients who met the SIRS criteria every day for the first 7 days of hospitalization were considered to have a sustained SIRS.

\section{Statistical Analysis}

We assessed the distribution and frequency of all demographic and clinical variables. Covariates were compared between those who were shunt dependent and those who did not require shunt placement using the chi-square goodness-of-fit test, Student t-test, or Wilcoxon rank-sum test. To determine which variables were associated with shunt dependence, logistic regression analyses were performed. All clinically relevant variables with $\mathrm{p}$ values < 0.20 in univariate analyses were included in a final multivariate logistic regression model. Variables that were dichotomized for analysis included: age $\geq 55$ years, MFS score $\geq 3$ ( 3 and 4 grouped due to small cell sizes), WFNS grade $\geq 3(3,4$, and 5 grouped due to small cell sizes), none/mild vasospasm versus moderate/severe vasospasm, and a sustained SIRS response. We assessed for interactions among all independent variables in the final model. To evaluate the respective contributions of the individual predictors of shunt dependence, we compared changes to the likelihood ratio value of the model (serving as an approximation of overall fit) after addition of each selected significant predictor. A receiver operating characteristic (ROC) analysis was performed to measure the predictive power of the final model. ${ }^{25}$ All analyses were conducted with STATA/SE (version 14.2 for Mac, StataCorp LLC). A $p$ value $<0.05$ was considered statistically significant.

\section{Results}

\section{Demographic and Clinical Characteristics}

Of the 244 patients initially identified during the study period, 193 were eligible and included in the final analysis (Fig. 1). The majority of patients were female (69\%) with a mean age of $54.6 \pm 12.7$ years. Patients most frequently had an MFS score of $3(60 \%)$, while 39\% of patients had a WFNS grade $\geq 3$. Anterior cerebral artery aneurysmsincluding $\mathrm{A}_{1}$, anterior communicating artery, $\mathrm{A}_{2}$, and pericallosal aneurysms - were the most common (39\%). Overall, 55\% of patients underwent surgical clip placement for initial treatment of their aneurysm.

\section{Shunt Dependence}

Patient characteristics grouped according to shunt dependence are presented in Table 1. Twenty-two percent of patients who had acute hydrocephalus requiring external CSF drainage later required shunt placement; $9.7 \%$ of shunt-dependent patients (comprising $1.6 \%$ of all study patients) required postdischarge shunt placement after readmission for symptoms of hydrocephalus. On bivariate analysis, MFS score $(\mathrm{p}=0.020)$, vasospasm severity $(\mathrm{p}=$ 0.001 ), acute hydrocephalus ( $\mathrm{p}<0.001$ ), and a sustained SIRS $(p=0.004)$ were significantly associated with shunt dependence. WFNS grade $(\mathrm{p}=0.108)$, aneurysm location $(\mathrm{p}=0.657)$, and mode of treatment $(\mathrm{p}=0.878)$ were not significantly associated with shunt dependence. Shunt dependence was not associated with discharge disposition: $42 \%$ of shunt-dependent patients were discharged home (including the 3 patients with shunt placement in a delayed fashion), compared to $48 \%$ of non-shunt-dependent patients $(\mathrm{p}=0.526)$.

\section{Sustained Systemic Inflammatory Response Syndrome}

Eighteen percent of all patients had a sustained SIRS spanning the first week of hospitalization. Twenty-one percent of patients with acute hydrocephalus during their ICU stay experienced a sustained SIRS $(p=0.072)$. A sustained SIRS was observed in 35\% of shunt-dependent patients versus $14 \%$ of those who did not require a shunt $(\mathrm{p}$ $=0.004)$. Our final logistic regression model included the following variables: female sex, hypertension, MFS score $\geq 3$, WFNS grade $\geq 3$, moderate to severe vasospasm, DND, acute hydrocephalus, and sustained SIRS. On multivariate logistic regression, female sex (OR $0.35,95 \%$ CI 0.142-0.885), moderate to severe vasospasm (OR 3.78, 95\% CI 1.333-10.745), acute hydrocephalus (OR 21.39, 95\% CI 2.260-202.417), and sustained SIRS (OR 2.94, 95\% CI 1.125-7.689) were significantly associated with shunt dependence after aneurysmal subarachnoid hemorrhage (Table 2). ROC analysis revealed an area under the curve (AUC) of 0.83 for the final regression model (Fig. 2).

\section{Discussion}

We identified several independent predictors for shunt dependence in a consecutive cohort of patients with aneurysmal subarachnoid hemorrhage. Patients with moderate to severe vasospasm, acute hydrocephalus, and a sustained SIRS had increased odds of shunt dependency. Females had lower odds of shunt-dependent hydrocephalus. These findings were independent of clinical and radiological grades of injury and hemorrhage severity.

Our $16 \%$ rate of shunt dependence is consistent with past reports, such as the large retrospective study by O'Kelly et al., which cited an $18.75 \%$ rate of shunt dependence following aneurysmal subarachnoid hemorrhage in 3120 patients. ${ }^{28}$ Prior studies have cited the relationship 


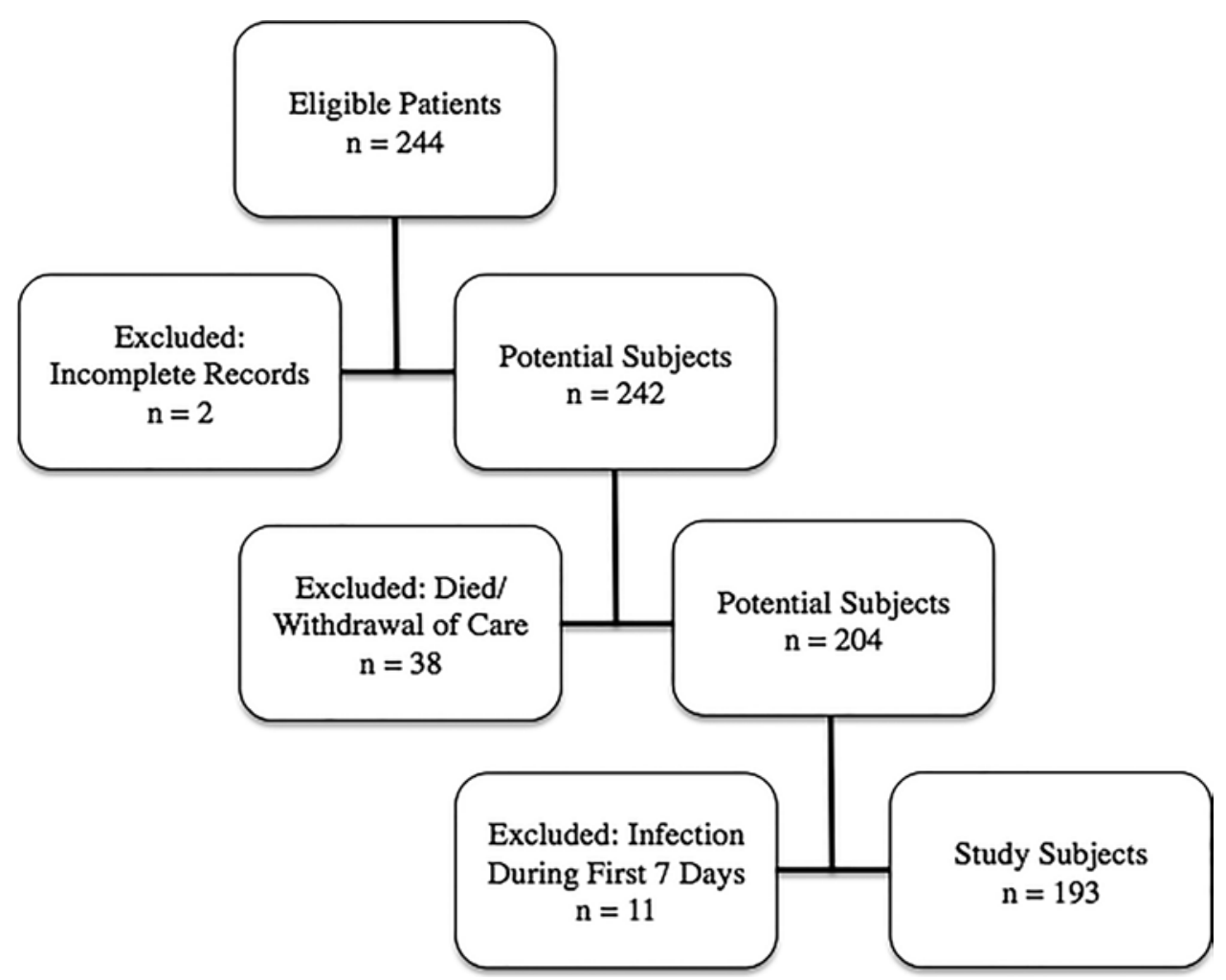

FIG. 1. Flow chart outlining patient selection criteria leading to 193 included study patients.

between shunt-dependent hydrocephalus and the following factors: age, sex, poor clinical grade, large amounts of subarachnoid and intraventricular hemorrhage (e.g., MFS scores of 3 or 4), acute hydrocephalus, posterior circulation aneurysms, early infarction, admission glucose, nosocomial meningitis, and intraparenchymal hemorrhage, among others. 5,14,28,29,36,39,40 However, after accounting for a sustained SIRS, traditional factors associated with shuntdependent hydrocephalus, such as MFS score and poor clinical grade, no longer remained statistically significant. WFNS grade approached significance with a $p$ value of 0.108 on univariate analysis, but did not remain significant on multivariate logistic regression. Patients who developed a DND had increased odds of shunt-dependent hydrocephalus on univariate analysis, but this was not significant after adjustment for other factors.

Acute hydrocephalus is a cause of early brain injury following subarachnoid hemorrhage and is often considered to be a form of noncommunicating, or obstructive, hydrocephalus due to the presence of intracisternal or intraventricular blood products limiting the outflow of CSF from the cranial vault. ${ }^{10}$ However, alternative causes of hydrocephalus have been described. MRI studies demonstrate that chronic hydrocephalus is often a communicating form, ${ }^{30,31}$ while some cases of acute hydrocephalus develop in the absence of significant intraventricular or cisternal hemorrhage. Animal studies have suggested that CSF hypersecretion resulting from dysfunctional choroid plexus may be implicated in the formation of hydrocephalus, particularly in the early stages after hemor- rhage. ${ }^{1,19}$ However, Kanat et al. suggest that increased CSF production is not a likely cause of hydrocephalus in the late stages following hemorrhage due to degeneration of the choroid plexus; thus, alternative mechanisms such as fibrosis of arachnoid granulations and impaired CSF reabsorption may contribute to chronic communicating hydrocephalus. ${ }^{16}$

Fibrosis of arachnoid granulations and impaired CSF reabsorption causing a communicating hydrocephalus has been attributed to a localized inflammatory reaction following subarachnoid hemorrhage. ${ }^{34,43}$ CSF levels of interleukin-6 are increased after subarachnoid hemorrhage and have been independently associated with shunt dependence. ${ }^{14,42}$ Thrombin, which is increased in the CSF following subarachnoid hemorrhage, has been tied to an increase in TGF- $\beta 1$ expression and a resultant proinflammatory and proliferative response in the meninges causing arachnoid granulation fibrosis. ${ }^{23}$

Simard et al. found that intraventricular hemorrhage sufficient to cause hydrocephalus was associated with a significant increase in NF- $\mathrm{KB}$ signaling within the CSF barrier cells of the choroid plexus and ependymal lining. ${ }^{32}$ These authors also noted an increase in immunoglobulin $\mathrm{G}$ uptake by choroid plexus cells suggestive of a potential increase in transcellular passage of protein that could alter characteristics of the CSF produced, resulting in an increase in protein content. ${ }^{32} \mathrm{~A}$ study by Lattke et al. similarly linked NF- $\mathrm{KB}$ signaling and hydrocephalus, demonstrating that inhibition of $\kappa \mathrm{B}$ kinase (IKK)/NF$\kappa \mathrm{B}-$ induced neuroinflammation interfered with normal 
TABLE 1. Characteristics of 193 patients admitted with aneurysmal subarachnoid hemorrhage grouped according to shunt dependence

\begin{tabular}{|c|c|c|c|}
\hline Characteristic & $\begin{array}{c}\text { Shunt } \\
\text { Dependent } \\
(n=31)\end{array}$ & $\begin{array}{l}\text { No Shunt } \\
(n=162)\end{array}$ & $\begin{array}{c}\mathrm{p} \\
\text { Value }\end{array}$ \\
\hline Mean age \pm SD (yrs) & $52.94 \pm 12.46$ & $54.88 \pm 12.76$ & 0.437 \\
\hline Female, $\mathrm{n}(\%)$ & $17(55)$ & $116(72)$ & 0.065 \\
\hline MFS score, $n(\%)$ & & & 0.020 \\
\hline 0 & $1(3)$ & $3(2)$ & \\
\hline 1 & $2(6)$ & $31(19)$ & \\
\hline 2 & $0(0)$ & $1(0.6)$ & \\
\hline 3 & $15(48)$ & $100(62)$ & \\
\hline 4 & $13(42)$ & $27(17)$ & \\
\hline WFNS Grade, n (\%) & & & 0.108 \\
\hline 1 & $5(16)$ & $63(39)$ & \\
\hline 2 & $8(26)$ & $42(26)$ & \\
\hline 3 & $2(6)$ & $7(4)$ & \\
\hline 4 & $11(35)$ & $37(23)$ & \\
\hline 5 & $5(16)$ & $13(8)$ & \\
\hline Aneurysm location, $\mathrm{n}(\%)$ & & & 0.657 \\
\hline $\mathrm{ACA}^{*}$ & $15(48)$ & $61(38)$ & \\
\hline ICA† & $9(29)$ & $54(33)$ & \\
\hline MCA & $4(13)$ & $21(13)$ & \\
\hline PC & $3(10)$ & $26(16)$ & \\
\hline Mode of treatment, $\mathrm{n}(\%)$ & & & 0.878 \\
\hline Noneł & $0(0)$ & $3(2)$ & \\
\hline Surgical clipping & $18(58)$ & $88(54)$ & \\
\hline Endovascular & $13(42)$ & $71(44)$ & \\
\hline Vasospasm severity, n (\%) & & & 0.001 \\
\hline None & $10(32)$ & $73(45)$ & \\
\hline Mild & $0(0)$ & $34(21)$ & \\
\hline Moderate & $11(35)$ & $25(15)$ & \\
\hline Severe & $10(32)$ & $30(19)$ & \\
\hline DND, n (\%) & $13(42)$ & $32(20)$ & 0.007 \\
\hline Infarct, n (\%) & $7(23)$ & $33(20)$ & 0.781 \\
\hline Acute hydrocephalus, $\mathrm{n}(\%) \S$ & $30(97)$ & $104(64)$ & $<0.001$ \\
\hline $\begin{array}{l}\text { Mean length of stay } \pm \text { SD } \\
\text { (days) }\end{array}$ & $33.68 \pm 14.89$ & $20.93 \pm 11.22$ & $<0.001$ \\
\hline Sustained SIRS, n (\%) & $11(35)$ & $23(14)$ & 0.004 \\
\hline Discharge disposition, n (\%) & & & 0.526 \\
\hline Home & 13 (42)爪 & $78(48)$ & \\
\hline Other & $18(58)$ & $84(52)$ & \\
\hline
\end{tabular}

$\mathrm{ACA}=$ anterior cerebral artery; ICA = internal carotid artery; $\mathrm{MCA}=$ middle cerebral artery; $P C=$ posterior circulation.

${ }^{*}$ Includes $A_{1}$, anterior communicating, $A_{2}$, and pericallosal aneurysms. $\dagger$ Includes anterior choroidal, posterior communicating, and ICA terminus aneurysms.

$\ddagger$ Three patients did not require surgical or endovascular treatment because their cerebral aneurysms, which were originally seen on angiographic study, were no longer visualized on subsequent angiography, presumably due to thrombosis.

$\S$ Acute hydrocephalus refers to symptomatic hydrocephalus requiring external CSF drainage during inpatient stay.

II Includes 3 patients who were initially discharged home and subsequently readmitted for delayed treatment of chronic hydrocephalus.
TABLE 2. Adjusted odds ratios of shunt dependence among patients admitted with aneurysmal subarachnoid hemorrhage

\begin{tabular}{lrcc}
\hline \multicolumn{1}{c}{ Variable } & $\begin{array}{c}\text { Adjusted } \\
\text { OR }\end{array}$ & $\begin{array}{c}95 \% \\
\mathrm{Cl}\end{array}$ & $\begin{array}{c}\mathrm{p} \\
\text { Value }\end{array}$ \\
\hline Female sex & 0.35 & $0.142-0.885$ & 0.026 \\
\hline Hypertension & 2.66 & $0.974-7.255$ & 0.056 \\
\hline MFS score $\geq 3$ & 0.32 & $0.062-1.631$ & 0.170 \\
\hline WFNS grade $\geq 3$ & 1.02 & $0.398-2.635$ & 0.961 \\
\hline Moderate to severe vasospasm & 3.78 & $1.333-10.745$ & 0.012 \\
\hline DND & 1.68 & $0.595-4.733$ & 0.328 \\
\hline Acute hydrocephalus* & 21.39 & $2.260-202.417$ & 0.008 \\
\hline Sustained SIRS & 2.94 & $1.125-7.689$ & 0.028 \\
\hline
\end{tabular}

* Acute hydrocephalus refers to symptomatic hydrocephalus requiring external CSF drainage during inpatient stay.

ependymal ciliogenesis and was associated with the development of hydrocephalus. ${ }^{22}$ More recently, Karimy et al. demonstrated that intraventricular hemorrhage causes hydrocephalus through TLR4-NF- $\mathrm{KB}$-mediated inflammation and upregulation of the pSPAK-pNKCC1 complex, producing CSF hypersection from the choroid plexus epithelium. ${ }^{19}$

Prior studies reveal that altering the localized inflammatory response following subarachnoid and intraventricular hemorrhage by inhibiting the TGF- $\beta 1 / \mathrm{Smad} /$ CTGF pathway or activating the cannabinoid receptor-2 pathway may alleviate chronic hydrocephalus. ${ }^{34,43}$ Karimy et al. also showed that several pharmacological or genetic approaches are capable of reducing CSF hypersecretion, including NF- $\mathrm{KB}$ inhibition with ammonium pyrrolidinedithiocarbamate, SPAK inhibition with closantel, treatment with STOCK1S-50699 (which disrupts SPAK and NKCC1 binding), and treatment with bumetanide, an inhibitor of NKCC1-dependent ion transport. TAK-242 or $T L R 4$ gene deletion is also capable of significantly reducing CSF secretion relative to controls. ${ }^{19}$

These findings suggest that an ability to limit localized inflammatory responses following subarachnoid hemorrhage may have the potential to reduce the incidence or duration of hydrocephalus. ${ }^{8}$ A reduction in shunt dependency may have several beneficial effects, particularly when considering the complications associated with prolonged EVD weaning and shunt placement, such as catheter- or shunt-related meningitis, intracerebral hemorrhage, and the limited longevity of shunt hardware necessitating surgical revision. ${ }^{17,20,21,28}$

Our study evaluated the relationship between systemic inflammation and shunt-dependent hydrocephalus, as opposed to the previously studied localized inflammatory responses contained within the CNS. In initial studies SIRS has been linked to higher WFNS grades and Fisher scores, likely representing the severity of a patient's underlying injury. ${ }^{4,33,44}$ Recent studies have demonstrated that extracerebral organ dysfunction can exist concurrently with acute brain injury following aneurysmal subarachnoid hemorrhage. ${ }^{8}$ Proposed mechanisms for concurrent derangements in cerebral and systemic physiology include direct 




FIG. 2. ROC curve for final regression model predicting shunt dependence (AUC $=0.8344)$. Figure is available in color online only.

CNS modulation of extracerebral organ systems, alteration of host immune responses, and a possible predisposition to a heightened state of systemic inflammation. ${ }^{11,26,27}$ While neurological injury has been proven to alter blood-brain barrier function, a previous report failed to show a direct correlation between serum and CSF cytokine levels following subarachnoid hemorrhage. ${ }^{9,38}$ Unfortunately, our study does not provide further characterization of the potential causal relationship between systemic inflammation and shunt-dependent hydrocephalus. However, we have successfully demonstrated an association between chronic shunt-dependent hydrocephalus and the following variables: acute hydrocephalus, cerebral vasospasm, and sustained SIRS.

A limitation of our study is its retrospective nature, making it prone to incomplete data collection and inherent bias. However, our inclusion of a consecutive cohort of patients over a 4-year timespan may reduce selection bias. While our study cohort is composed of a reasonable sample size of 193 patients, we chose to pool certain categorical variables for analysis due to small cell sizes. Our SIRS data were compiled from the first 7 days of hospitalization, which represents a particular advantage over prior studies of SIRS in subarachnoid hemorrhage that were limited to the first 4 days of hospital stay. ${ }^{4,36,44}$ Future studies will be required to evaluate the time course and impact of SIRS on other forms of secondary brain injury and the potential therapeutic implications.

\section{Conclusions}

Sustained SIRS was found to be a predictor of shuntdependent hydrocephalus following aneurysmal subarach- noid hemorrhage even after adjustment for other factors in a multivariate logistic regression model. Moderate to severe vasospasm and acute hydrocephalus were also independently associated with increased odds of shunt dependency. Patients with acute hydrocephalus and sustained SIRS may be candidates for early shunt placement due to the high likelihood of developing shunt-dependent hydrocephalus. Our report reinforces the need for further study of the relationship between the systemic inflammatory response and secondary brain injury following subarachnoid hemorrhage. Components of the local or systemic inflammatory response may serve as potential targets for future therapies in patients with subarachnoid hemorrhage.

\section{References}

1. Banizs B, Pike MM, Millican CL, Ferguson WB, Komlosi P, Sheetz J, et al: Dysfunctional cilia lead to altered ependyma and choroid plexus function, and result in the formation of hydrocephalus. Development 132:5329-5339, 2005

2. Bartlett JG, Gerding DN: Clinical recognition and diagnosis of Clostridium difficile infection. Clin Infect Dis 46 (Suppl 1):S12-S18, 2008

3. Connolly ES Jr, Rabinstein AA, Carhuapoma JR, Derdeyn CP, Dion J, Higashida RT, et al: Guidelines for the management of aneurysmal subarachnoid hemorrhage: a guideline for healthcare professionals from the American Heart Association/American Stroke Association. Stroke 43:1711-1737, 2012

4. Dhar R, Diringer MN: The burden of the systemic inflammatory response predicts vasospasm and outcome after subarachnoid hemorrhage. Neurocrit Care 8:404-412, 2008

5. Erixon HO, Sorteberg A, Sorteberg W, Eide PK: Predictors of shunt dependency after aneurysmal subarachnoid hemor- 
rhage: results of a single-center clinical trial. Acta Neurochir (Wien) 156:2059-2069, 2014

6. Frontera JA, Claassen J, Schmidt JM, Wartenberg KE, Temes $\mathrm{R}$, Connolly ES Jr, et al: Prediction of symptomatic vasospasm after subarachnoid hemorrhage: the modified fisher scale. Neurosurgery 59:21-27, 2006

7. Frontera JA, Fernandez A, Schmidt JM, Claassen J, Wartenberg KE, Badjatia N, et al: Defining vasospasm after subarachnoid hemorrhage: what is the most clinically relevant definition? Stroke 40:1963-1968, 2009

8. Gruber A, Reinprecht A, Illievich UM, Fitzgerald R, Dietrich W, Czech T, et al: Extracerebral organ dysfunction and neurologic outcome after aneurysmal subarachnoid hemorrhage. Crit Care Med 27:505-514, 1999

9. Gruber A, Rössler K, Graninger W, Donner A, Illievich MU, Czech T: Ventricular cerebrospinal fluid and serum concentrations of sTNFR-I, IL-1ra, and IL- 6 after aneurysmal subarachnoid hemorrhage. J Neurosurg Anesthesiol 12:297-306, 2000

10. Güresir E, Schuss P, Borger V, Vatter H: Experimental subarachnoid hemorrhage: double cisterna magna injection rat model-assessment of delayed pathological effects of cerebral vasospasm. Transl Stroke Res 6:242-251, 2015

11. Hirashima Y, Nakamura S, Endo S, Kuwayama N, Naruse Y, Takaku A: Elevation of platelet activating factor, inflammatory cytokines, and coagulation factors in the internal jugular vein of patients with subarachnoid hemorrhage. Neurochem Res 22:1249-1255, 1997

12. Hooton TM, Bradley SF, Cardenas DD, Colgan R, Geerlings SE, Rice JC, et al: Diagnosis, prevention, and treatment of catheter-associated urinary tract infection in adults: 2009 International Clinical Practice Guidelines from the Infectious Diseases Society of America. Clin Infect Dis 50:625-663, 2010

13. Howell MD, Davis AM: Management of sepsis and septic shock. JAMA 317:847-848, 2017

14. Jabbarli R, Bohrer AM, Pierscianek D, Müller D, Wrede KH, Dammann P, et al: The CHESS score: a simple tool for early prediction of shunt dependency after aneurysmal subarachnoid hemorrhage. Eur J Neurol 23:912-918, 2016

15. Kalil AC, Metersky ML, Klompas M, Muscedere J, Sweeney DA, Palmer LB, et al: Management of adults with hospitalacquired and ventilator-associated pneumonia: 2016 clinical practice guidelines by the Infectious Diseases Society of America and the American Thoracic Society. Clin Infect Dis 63:e61-e111, 2016

16. Kanat A, Turkmenoglu O, Aydin MD, Yolas C, Aydin N, Gursan N, et al: Toward changing of the pathophysiologic basis of acute hydrocephalus after subarachnoid hemorrhage: a preliminary experimental study. World Neurosurg 80:390-395, 2013

17. Kanev PM, Sheehan JM: Reflections on shunt infection. Pediatr Neurosurg 39:285-290, 2003

18. Karimy JK, Duran D, Hu JK, Gavankar C, Gaillard JR, Bayri $\mathrm{Y}$, et al: Cerebrospinal fluid hypersecretion in pediatric hydrocephalus. Neurosurg Focus 41(5):E10, 2016

19. Karimy JK, Zhang J, Kurland DB, Theriault BC, Duran D, Stokum JA, et al: Inflammation-dependent cerebrospinal fluid hypersecretion by the choroid plexus epithelium in posthemorrhagic hydrocephalus. Nat Med 23:997-1003, 2017

20. Kassell NF, Torner JC, Haley EC Jr, Jane JA, Adams HP, Kongable GL: The International Cooperative Study on the Timing of Aneurysm Surgery. Part 1: Overall management results. J Neurosurg 73:18-36, 1990

21. Lai L, Morgan MK: Predictors of in-hospital shunt-dependent hydrocephalus following rupture of cerebral aneurysms. J Clin Neurosci 20:1134-1138, 2013

22. Lattke M, Magnutzki A, Walther P, Wirth T, Baumann B: Nuclear factor $\kappa \mathrm{B}$ activation impairs ependymal ciliogenesis and links neuroinflammation to hydrocephalus formation. J Neurosci 32:11511-11523, 2012

23. Li T, Zhang P, Yuan B, Zhao D, Chen Y, Zhang X: Thrombin-induced TGF- $\beta 1$ pathway: a cause of communicating hydrocephalus post subarachnoid hemorrhage. Int J Mol Med 31:660-666, 2013

24. Little AS, Zabramski JM, Peterson M, Goslar PW, Wait SD, Albuquerque FC, et al: Ventriculoperitoneal shunting after aneurysmal subarachnoid hemorrhage: analysis of the indications, complications, and outcome with a focus on patients with borderline ventriculomegaly. Neurosurgery 62:618627, 2008

25. Lu Q, Elston RC: Using the optimal receiver operating characteristic curve to design a predictive genetic test, exemplified with type 2 diabetes. Am J Hum Genet 82:641-651, 2008

26. Mathiesen T, Lefvert AK: Cerebrospinal fluid and blood lymphocyte subpopulations following subarachnoid haemorrhage. Br J Neurosurg 10:89-92, 1996

27. McKeating EG, Andrews PJ, Signorini DF, Mascia L: Transcranial cytokine gradients in patients requiring intensive care after acute brain injury. Br J Anaesth 78:520-523, 1997

28. O'Kelly CJ, Kulkarni AV, Austin PC, Urbach D, Wallace MC: Shunt-dependent hydrocephalus after aneurysmal subarachnoid hemorrhage: incidence, predictors, and revision rates. Clinical article. J Neurosurg 111:1029-1035, 2009

29. Rincon F, Gordon E, Starke RM, Buitrago MM, Fernandez A, Schmidt JM, et al: Predictors of long-term shunt-dependent hydrocephalus after aneurysmal subarachnoid hemorrhage. Clinical article. J Neurosurg 113:774-780, 2010

30. Saliou G, Balédent O, Lehmann P, Paradot G, Gondry-Jouet $\mathrm{C}$, Bouzerar R, et al: [Acute CSF changes in the mesencephalon aqueduct after subarachnoid hemorrhage as measured by PC-MRI.] J Neuroradiol 36:41-47, 2009 (Fr)

31. Saliou G, Paradot G, Gondry C, Bouzerar R, Lehmann P, Meyers ME, et al: A phase-contrast MRI study of acute and chronic hydrodynamic alterations after hydrocephalus induced by subarachnoid hemorrhage. J Neuroimaging 22:343-350, 2012

32. Simard PF, Tosun C, Melnichenko L, Ivanova S, Gerzanich V, Simard JM: Inflammation of the choroid plexus and ependymal layer of the ventricle following intraventricular hemorrhage. Transl Stroke Res 2:227-231, 2011

33. Tam AK, Ilodigwe D, Mocco J, Mayer S, Kassell N, Ruefenacht D, et al: Impact of systemic inflammatory response syndrome on vasospasm, cerebral infarction, and outcome after subarachnoid hemorrhage: exploratory analysis of CONSCIOUS-1 database. Neurocrit Care 13:182-189, 2010

34. Tan Q, Chen Q, Feng Z, Shi X, Tang J, Tao Y, et al: Cannabinoid receptor 2 activation restricts fibrosis and alleviates hydrocephalus after intraventricular hemorrhage. Brain Res 1654 (Pt A): 24-33, 2017

35. Teasdale GM, Drake CG, Hunt W, Kassell N, Sano K, Pertuiset B, et al: A universal subarachnoid hemorrhage scale: report of a committee of the World Federation of Neurosurgical Societies. J Neurol Neurosurg Psychiatry 51:1457, 1988

36. Tso MK, Ibrahim GM, Macdonald RL: Predictors of shuntdependent hydrocephalus following aneurysmal subarachnoid hemorrhage. World Neurosurg 86:226-232, 2016

37. Tunkel AR, Hasbun R, Bhimraj A, Byers K, Kaplan SL, Scheld WM, et al: 2017 Infectious Diseases Society of America's clinical practice guidelines for healthcare-associated ventriculitis and meningitis. Clin Infect Dis 64:e34-e65, 2017

38. Varatharaj A, Galea I: The blood-brain barrier in systemic inflammation. Brain Behav Immun 60:1-12, 2017

39. Walcott BP, Iorgulescu JB, Stapleton CJ, Kamel H: Incidence, timing, and predictors of delayed shunting for hydrocephalus after aneurysmal subarachnoid hemorrhage. Neurocrit Care 23:54-58, 2015 
40. Wang YM, Lin YJ, Chuang MJ, Lee TH, Tsai NW, Cheng $\mathrm{BC}$, et al: Predictors and outcomes of shunt-dependent hydrocephalus in patients with aneurysmal sub-arachnoid hemorrhage. BMC Surg 12:12, 2012

41. Weidauer S, Lanfermann H, Raabe A, Zanella F, Seifert V, Beck J: Impairment of cerebral perfusion and infarct patterns attributable to vasospasm after aneurysmal subarachnoid hemorrhage: a prospective MRI and DSA study. Stroke 38:1831-1836, 2007

42. Wostrack M, Reeb T, Martin J, Kehl V, Shiban E, Preuss A, et al: Shunt-dependent hydrocephalus after aneurysmal subarachnoid hemorrhage: the role of intrathecal interleukin-6. Neurocrit Care 21:78-84, 2014

43. Yan H, Chen Y, Li L, Jiang J, Wu G, Zuo Y, et al: Decorin alleviated chronic hydrocephalus via inhibiting TGF- $\beta 1 / \mathrm{Smad} /$ CTGF pathway after subarachnoid hemorrhage in rats. Brain Res 1630:241-253, 2016

44. Yoshimoto Y, Tanaka Y, Hoya K: Acute systemic inflammatory response syndrome in subarachnoid hemorrhage. Stroke 32:1989-1993, 2001

45. Zaidi HA, Montoure A, Elhadi A, Nakaji P, McDougall CG, Albuquerque FC, et al: Long-term functional outcomes and predictors of shunt-dependent hydrocephalus after treatment of ruptured intracranial aneurysms in the BRAT trial: revisiting the clip vs coil debate. Neurosurgery 76:608-614, 2015

\section{Disclosures}

Dr. Jindal reports receiving research grant funding from Stryker Neurovascular.

\section{Author Contributions}

Conception and design: Wessell, Kole, Simard. Acquisition of data: Wessell, Kole, Cannarsa, Oliver. Analysis and interpretation of data: all authors. Drafting the article: all authors. Critically revising the article: all authors. Reviewed submitted version of manuscript: all authors. Approved the final version of the manuscript on behalf of all authors: Wessell. Statistical analysis: Wessell. Study supervision: Wessell, Jindal, Miller, Gandhi, Parikh, Badjatia, Aldrich, Simard.

\section{Correspondence}

Aaron P. Wessell: University of Maryland School of Medicine, Baltimore, MD. awessell@som.umaryland.edu. 